TRANSACTIONS OF THE

AMERICAN MATHEMATICAL SOCIETY

Volume 300, Number 2, April 1987

\title{
A GENERALIZED FATOU THEOREM
}

\author{
B. A. MAIR AND DAVID SINGMAN
}

\begin{abstract}
In this paper, a general Fatou theorem is obtained for functions which are integrals of kernels against measures on $\mathbf{R}^{n}$. These include solutions of Laplace's equation on an upper half-space, parabolic equations on an infinite slab and the heat equation on a right half-space. Lebesgue almost everywhere boundary limits are obtained within regions which contain sequences approaching the boundary with any prescribed degree of tangency.
\end{abstract}

0. Introduction. It is well known that every positive solution of Laplace's equation on $\mathbf{R}^{n} \times \mathbf{R}_{+}$has finite nontangential limits Lebesgue almost everywhere on $\mathbf{R}^{n}$. Recently, this result has been improved by A. Nagel and E. Stein (cf. [10]) to include limits within regions which allow sequential approach with any degree of tangency to the boundary, $\mathbf{R}^{n}$. These regions are constructed by taking a countable union of cones with vertices on a surface which is tangential to $\mathbf{R}^{n}$.

In this paper we consider $\mathbf{R}^{n}$ equipped with a translation-invariant pseudodistance, $\rho$, and define the analogue of a cone when distance is measured by $\rho$ instead of the Euclidean norm (these are the "standard" sets). The " $\alpha$-admissible" sets are the analogues of the sets which satisfy "a cone condition with aperture $\alpha$ " (cf. [10]). By using the maximal function techniques in $[\mathbf{1 0}]$ we obtain a differentiation theorem relative to the $\rho$-balls.

In $\S 2$, we obtain a general Fatou theorem for functions of the form

$$
\int K(x, t ; y) d \mu(y)
$$

on $\mathbf{R}_{+}^{n+1}$, where the conditions on $K$ are stated in terms of $\rho$.

This theorem is applied in $\S 3$ to obtain the result of Nagel and Stein for Laplace's equation on $\mathbf{R}_{+}^{n+1}$ and analogous results for parabolic equations on $\mathbf{R}^{n} \times(0, T)$ (resp. the heat equation on $\mathbf{R}^{n-1} \times \mathbf{R}_{+} \times(-\infty, T)$ ), where the cone is replaced by a parabolic region (resp. two-sided parabolic region).

We conclude by showing that under certain additional conditions on $K$, the $\alpha$-admissible regions are best in some sense.

We take this opportunity to thank Stanton Philipp for the stimulating discussions we had and J. Chabrowski for suggesting that the result of Nagel and Stein may be extended to parabolic equations on $\mathbf{R}^{n} \times(0, T)$.

Received by the editors January 15, 1986. Presented to the 92nd Annual Meeting of the AMS, January 7, 1986.

1980 Mathematics Subject Classification (1985 Revision). Primary 31B25; Secondary 35J05, 35K05, 35K20, 42B25.

Key words and phrases. Fatou theorem, Laplace equation, maximal function, weak type, pseudo-distance, parabolic equation, $\alpha$-admissible, $\Omega$-Lebesgue set.

(C) 1987 American Mathematical Society $0002-9947 / 87 \$ 1.00+\$ .25$ per page 
1. A differentiation theorem. Throughout this paper, the Lebesgue measure of $E \subset \mathbf{R}^{n}$ will be denoted by $|E|$ and $\gamma \geq 1$ is a fixed constant. $C$ denotes a general constant which may depend on $n, \gamma$ and other constants and is not necessarily the same at different occurrences.

$\rho: \mathbf{R}^{n} \times \mathbf{R}^{n} \rightarrow[0, \infty)$ is assumed to satisfy the following properties:

(1.1) For all $x, y, z \in \mathbf{R}^{n}$,

(i) $\rho(x, y)=\rho(y, x)$,

(ii) $\rho(x, y)=0 \Leftrightarrow x=y$,

(iii) $\rho(x+z, y+z)=\rho(x, y)$,

(iv) $\rho(x, y) \leq \gamma[\rho(x, z)+p(z, y)]$.

That is, $\rho$ is a translation-invariant pseudo-distance (cf. [3]).

(1.2) For each $x \in \mathbf{R}^{n}$ and $r>0$ define $B(x, r)=\{y: \rho(x, y)<r\}$, the $\rho$-ball with center $x$ and radius $r$.

(i) $\{B(x, r): r>0\}$ forms a base for the open neighborhoods of $x$

(in the Euclidean topology).

(ii) For each $\alpha>0$ there exists $\tau_{n}(\alpha)$ such that

$$
|B(0, \alpha, r)| \leq C \tau_{n}(\alpha)|B(0, r)|
$$

for all $r>0$.

Observe that, by translation-invariance of $\rho$, we can replace 0 by any $x \in \mathbf{R}^{n}$ in the above inequality.

The examples of $\rho$ that will be used in $\S 3$ are

(a) $\rho(x, y)=|x-y|$ (Euclidean metric),

(b) $\rho(x, y)=|x-y|^{2}$,

(c) $\rho(x, y)=\left(\left|x^{\prime}-y^{\prime}\right|^{2}+\left|x_{n}-y_{n}\right|\right)^{1 / 2}$ where $x=\left(x^{\prime}, x_{n}\right)$ and $y=\left(y^{\prime}, y_{n}\right) \in$ $\mathbf{R}^{n-1} \times \mathbf{R}$.

In (a) $\rho$ is a metric, $|B(0, r)|=C r^{n}$ and $\tau_{n}(\alpha)=\alpha^{n}$. In (b), $\gamma=2,|B(0, r)|=$ $C r^{n / 2}$ and $\tau_{n}(\alpha)=\alpha^{n / 2}$. In (c), $\rho$ is a metric (see $\S 3$ ), $|B(0, r)|=C r^{n+1}$ and $\tau_{n}(\alpha)=\alpha^{n+1}$.

For each $\alpha>0$ and $y \in \mathbf{R}^{n}$, define the $\alpha$-standard region with vertex $y$ by

$$
A(y ; \alpha)=\left\{(x, t) \in \mathbf{R}^{n} \times \mathbf{R}_{+}: \rho(x, y)<\alpha t\right\} .
$$

Then, for each $t>0$,

$$
|\{x:(x, t) \in A(y ; \alpha)\}|=|B(y, \alpha t)| \leq C \tau_{n}(\alpha)|B(0, t)| .
$$

Definition 1.3. Let $\Omega \subset \mathbf{R}_{+}^{n+1}$ be open and $\alpha>0$.

(1) For each $t>0$, define $\Omega(t)=\left\{x \in \mathbf{R}^{n}:(x, t) \in \Omega\right\}, Q(t)=\bigcup_{x \in \Omega(t)} B(x, t)$.

(2) $\Omega$ is said to be $\alpha$-admissible if

(a) $0 \in \Omega(t)$, for all $t>0$,

(b) $0<s<t \Rightarrow \Omega(s) \subset \Omega(t)$,

(c) there exists open $\Omega^{\prime} \supset \Omega$ such that

(i) $\left|\Omega^{\prime}(t)\right| \leq C|B(0, t)|$, for all $t>0$, and

(ii) $(y, s) \in \Omega,(x, t-s) \in A(y ; \alpha) \Rightarrow(x, t) \in \Omega^{\prime}$.

In case $\rho$ is a metric, we define $\Omega^{\prime}=\Omega$ (cf. Proposition 1.12). In this case, (a) can be replaced by the condition $(0,0) \in \bar{\Omega}$. 
Note that every $\alpha$-standard region is $\alpha$-admissible.

Proceeding as in Lemma 1 of [10], one shows

LEMMA 1.4. Assume $\Omega$ is $\alpha$-admissible.

(i) For each $t>0, Q(t) \subset \Omega^{\prime}((\alpha+1) / \alpha \cdot t)$.

(ii) For each $t>0,|B(0, t)| \leq|Q(t)| \leq C \tau_{n}((\alpha+1) / \alpha)|B(0, t)|$.

(iii) There exists an integer $M=M(\alpha)$ such that for any $t>0$ there exist $x_{1}, x_{2}, \ldots, x_{M} \in \Omega(t)$ such that $Q(t) \subset \bigcup_{i=1}^{M} B\left(x_{i}, \gamma(2 \gamma+1) t\right)$.

Definition 1.5. Let $\Omega \subset \mathbf{R}_{+}^{n+1}$ have $(0,0)$ as a limit point. For each regular Borel measure $\mu$ on $\mathbf{R}^{n}$ and $y \in \mathbf{R}^{n}$, define

$$
\begin{aligned}
M_{\Omega} \mu(y) & =\sup _{(x, t) \in \Omega} \frac{\mu(B(y+x, t))}{|B(0, t)|} \\
M_{\Omega}^{*} \mu(y) & =\limsup _{\Omega \ni(x, t) \rightarrow 0} \frac{\mu(B(y+x, t))}{|B(0, t)|} \\
N \mu(y) & =\sup _{t>0} \frac{\mu(y+Q(t))}{|Q(t)|} .
\end{aligned}
$$

From Lemma 1.4 we see immediately

LEMMA 1.6. If $\Omega$ is $\alpha$-admissible and $\mu$ is a regular Borel measure on $\mathbf{R}^{n}$, then

$$
M_{\Omega}^{*} \mu(y) \leq M_{\Omega} \mu(y) \leq C \tau_{n}\left(\frac{\alpha+1}{\alpha}\right) N \mu(y), \quad \text { for all } y \in \mathbf{R}^{n} .
$$

LEMMA 1.7. Let $\Omega$ be $\alpha$-admissible. Then there is a constant $C>0$ such that for any finite Borel measure $\mu$ on $\mathbf{R}^{n}$,

$$
|\{x: N \mu(x)>\lambda\}| \leq C|\mu|_{1} / \lambda \text { for all } \lambda>0 .
$$

Proof. Let $\lambda>0$ and $E_{\lambda}=\{x: N \mu(x)>\lambda\}$. Then for each $x \in E_{\lambda}$ there exists $t(x)>0$ such that $\mu(x+Q(t(x)))>\lambda|Q(t(x))|$. Now, $0 \in \Omega(t(x)) \subset Q(t(x))$ hence $x \in x+Q(t(x))$ and $E_{\lambda} \subset \bigcup_{x \in E_{\lambda}}(x+Q(t(x)))$. Let $F \subset E_{\lambda}$ be compact. Then there is a finite subcover $\left\{x_{j}+Q\left(t_{j}\right): j=1,2, \ldots, N\right\}$ where $t_{j}=t\left(x_{j}\right)$.

As in [10, pp. 90-91], we select a subcollection, $\left\{x_{j_{s}}+Q\left(t_{j_{s}}\right): s=1,2, \ldots, p\right\}$ with the following properties:

(a) the sets are pairwise disjoint,

(b) if $j \in\{1,2, \ldots, N\} \backslash\left\{j_{1}, j_{2}, \ldots, j_{p}\right\}$, there exists $s \in\{1,2, \ldots, p\}$ such that $\left(x_{j}+Q\left(t_{j}\right)\right) \cap\left(x_{j_{s}}+Q\left(t_{j_{s}}\right)\right) \neq \varnothing$ and $t_{j} \leq t_{j_{s}}$. Now from Lemma 1.4(iii), there is an integer $M>0$ such that for each $t>0$, there exists $v_{1}(t), v_{2}(t), \ldots, v_{M}(t)$ in $\Omega(t)$ such that $Q(t) \subset \bigcup_{i=1}^{M} B\left(v_{i}(t), \gamma(2 \gamma+1) t\right)$. Let

$$
\tilde{Q}(t)=\bigcup_{j, k=1}^{M}\left[v_{j}(t)-v_{k}(t)+\bigcup_{i=1}^{M} B\left(v_{i}(t), \gamma^{2}(2 \gamma+1)^{2} t\right)\right] \text {. }
$$

Then $|\tilde{Q}(t)| \leq C|B(0, t)|$ and $Q(t) \subset \tilde{Q}(t)$.

Now, suppose $\left(x_{j}+Q\left(t_{j}\right)\right) \cap\left(x_{j_{s}}+Q\left(t_{j_{s}}\right)\right) \neq \varnothing, t_{j} \leq t_{j_{s}}$. We claim that $x_{j}+Q\left(t_{j}\right) \subset x_{j_{s}}+\tilde{Q}\left(t_{j_{s}}\right)$. By assumption, there exist $a \in Q\left(t_{j}\right)$ and $b \in Q\left(t_{j_{s}}\right)$ such that $x_{j}+a=x_{j_{s}}+b$. Since $Q\left(t_{j}\right) \subset Q\left(t_{j_{s}}\right)$, for any $y \in Q\left(t_{j}\right)$ we can choose 
$v_{i}=v_{i}\left(t_{j_{s}}\right), v_{k}=v_{k}\left(t_{j_{s}}\right), v_{m}=v_{m}\left(t_{j_{s}}\right)$ in $\Omega\left(t_{j_{s}}\right)$ such that $\rho\left(y, v_{i}\right), \rho\left(a, v_{k}\right)$ and $\rho\left(b, v_{m}\right)<\gamma(2 \gamma+1) t_{j_{s}}$. Then,

$$
x_{j}+y-x_{j_{s}}=y+b-a=v_{m}-v_{k}+v_{i}+\left(y-v_{i}\right)+\left(b-v_{m}\right)+\left(v_{k}-a\right)
$$

and

$$
\begin{aligned}
\rho\left(\left[\left(y-v_{i}\right)+\left(b-v_{m}\right)+\left(v_{k}-a\right)\right], 0\right) & \leq \gamma\left\{\gamma\left[\rho\left(y, v_{i}\right)+\rho\left(b, v_{m}\right)\right]+\rho\left(a, v_{k}\right)\right\} \\
& <\gamma^{2}(2 \gamma+1)^{2} t_{j_{s}}
\end{aligned}
$$

which proves the claim.

It follows that $F \subset \bigcup_{s=1}^{p}\left(x_{j_{s}}+\tilde{Q}\left(t_{j_{s}}\right)\right)$. Thus

$$
\begin{aligned}
|F| & \leq \sum_{s=1}^{p}\left|\tilde{Q}\left(t_{j_{s}}\right)\right| \leq C \sum_{s=1}^{p}\left|B\left(0, t_{j_{s}}\right)\right| \leq C \sum_{s=1}^{p}\left|Q\left(t_{j_{s}}\right)\right| \\
& <\frac{C}{\lambda} \sum_{s=1}^{p} \mu\left(x_{j_{s}}+Q\left(t_{j_{s}}\right)\right) \leq \frac{C}{\lambda}|\mu|_{1} .
\end{aligned}
$$

The following result is immediate.

THEOREM 1.8. Let $\Omega$ be $\alpha$-admissible. Then, there is a constant $C>0$ such that for any finite measure $\mu$ on $\mathbf{R}^{n}$,

$$
\left|\left\{x: M_{\Omega} \mu(x)>\lambda\right\}\right| \leq C|\mu|_{1} / \lambda, \quad\left|\left\{x: M_{\Omega}^{*} \mu(x)>\lambda\right\}\right| \leq C|\mu|_{1} / \lambda
$$

for all $\lambda>0$.

We now obtain the following differentiation theorem by a slight variation of the standard method (cf. [11]).

THEOREM 1.9. Let $\Omega$ be $\alpha$-admissible and $\mu$ a signed, regular Borel measure on $\mathbf{R}^{n}$. Then

$$
\lim _{\Omega \ni(x, t) \rightarrow 0} \frac{\mu(B(y+x, t))}{|B(0, t)|}=f(y)
$$

for Lebesgue a.e. $y \in \mathbf{R}^{n}$, where $f$ is the usual Radon-Nikodyn derivative of $\mu$.

PROOF. We need only consider the case of a finite (positive) measure $\mu$.

For any locally integrable $F$ and $x_{0} \in \mathbf{R}^{n}$, define

$$
\Lambda F\left(x_{0}\right)=\limsup _{\Omega \ni(x, t) \rightarrow 0}\left\{\frac{1}{|B(0, t)|} \int_{B\left(x_{0}+x, t\right)} F(y) d y\right\}-F\left(x_{0}\right) .
$$

Since the $\rho$-balls form a base for the Euclidean topology, if $F$ is continuous, $\Lambda F(x)=$ 0 for all $x \in \mathbf{R}^{n}$. Hence by Theorem 1.8 and the denseness of continuous functions in $L^{1}$, the result holds for absolutely continuous $\mu$.

Now, if $\mu$ is singular, we have a Borel set $E$ such that $\mu(E)=0$ and $\left|E^{c}\right|=0$. Fix $\varepsilon>0$ and choose open $V \supset E$ such that $\mu(V)<\varepsilon$. Then again by property (1.2), $\left.M_{\Omega}^{*} \mu\right|_{V}=0$ on $V$, hence,

$$
\lim _{\Omega \ni(x, t) \rightarrow 0} \frac{\mu(B(y+x, t))}{|B(0, t)|}=0 \quad \text { a.e. } y
$$

by applying Theorem 1.8 . 
As usual (cf. [11, p. 11]), we can strengthen Theorem 1.9 to obtain the derivative in the variational sense (cf. [4, p. 291]).

DEFINITION 1.10. (i) The variation measure of a signed measure $\mu$ will be denoted by $\|\mu\|$.

(ii) Let $\mu$ be a signed measure with Radon-Nikodym derivative $f$. A point $x_{0} \in \mathbf{R}^{n}$ is in the $\Omega$-Lebesgue set of $\mu$ if

$$
\lim _{\Omega \ni(x, t) \rightarrow 0} \frac{\left\|\mu-f\left(x_{0}\right) m\right\| B\left(x_{0}+x, t\right)}{|B(0, t)|}=0,
$$

where $m$ is Lebesgue measure on $\mathbf{R}^{n}$.

THEOREM 1.11. Let $\Omega$ be $\alpha$-admissible and $\mu$ a signed Borel measure on $\mathbf{R}^{n}$. Then Lebesgue a.e. $x_{0}$ is in the $\Omega$-Lebesgue set of $\mu$.

We now give examples of $\alpha$-admissible sets which are not contained in any $\beta$ standard set (cf. [10, Lemma 9]).

PROPOSITION 1.12. Let $\left(x_{k}, t_{k}\right)$ be a sequence in $\mathbf{R}_{+}^{n+1}$ such that $t_{k+1} \leq t_{k}$, $\lim _{k \rightarrow \infty} t_{k}=0$ and $\rho\left(0, x_{k+1}\right) \leq A t_{k}$ for some constant $A>0$ and all $k$. For any $\alpha>0$, let

$$
\begin{aligned}
\Omega & =\left\{(x, t): \rho\left(x, x_{k}\right)<\alpha\left(t-t_{k}\right) \text { for some } k\right\}, \\
\Omega^{\prime} & =\left\{(x, t): \rho\left(x, x_{k}\right)<\alpha \gamma\left(t-t_{k}\right) \text { for some } k\right\} .
\end{aligned}
$$

Then $\Omega$ is $\alpha$-admissible.

PROOF. Fix $t>0$ and let $N$ be the first index for which $t_{N}<t$. If $(x, t) \in \Omega^{\prime}$ then there is a $k$ such that $\rho\left(x, x_{k}\right)<\alpha \gamma\left(t-t_{k}\right)$. Hence $k \geq N$. So

$$
\Omega^{\prime}(t) \subset B\left(x_{N}, \alpha \gamma t\right) \cup \bigcup_{k=N}^{\infty} B\left(x_{k+1}, \alpha \gamma t\right) .
$$

Now, $k \geq N \Rightarrow \rho\left(0, x_{k+1}\right) \leq A t_{k} \leq A t_{N}<A t$ hence $\rho\left(x, x_{k+1}\right)<\alpha \gamma t \Rightarrow \rho(x, 0)<$ $\gamma(\alpha \gamma+A) t$. Thus

$$
\Omega^{\prime}(t) \subset B\left(x_{N}, \alpha \gamma t\right) \cup B(0, \gamma(\alpha \gamma+A) t)
$$

and so

$$
\left|\Omega^{\prime}(t)\right| \leq C|B(0, t)| \text {. }
$$

The other properties of $\Omega$ in Definition 1.3 are obvious.

Note that if $\rho$ is a metric, $\Omega^{\prime}=\Omega$.

Now choose $\left(x_{k}, t_{k}\right)$ satisfying the conditions of the proposition and such that $\rho\left(0, x_{k}\right) / t_{k} \rightarrow \infty$. For example, choose $0<t_{1}<1$ and put $t_{k+1}=\frac{1}{2} t_{k}^{2}$. Choose $x_{k}$ such that $\rho\left(0, x_{k}\right)=\sqrt{t_{k}}$. Then the region $\Omega$ generated by this sequence is not contained in any $\beta$-standard set $A(0, \beta)$. Indeed, suppose it were. Then $\left(x_{k}, 2 t_{k}\right) \in$ $\Omega$ for all $k$, hence $\rho\left(x_{k}, 0\right)$ would be less than $2 \beta t_{k}$ and so $\rho\left(x_{k}, 0\right) / t_{k}$ would be bounded, contradicting our construction.

By adapting the process in $[\mathbf{1 0}$, p. 98$]$ we show how to construct $\alpha$-admissible regions allowing sequential apporoach with any prescribed degree of tangency.

Let $\Psi:[0, \infty) \rightarrow[0, \infty]$ be such that $\Psi(0)=0, \lim _{\lambda \rightarrow 0^{+}} \Psi(\lambda) / \lambda=0$. We denote the $k$ th iterate of $\Psi$ by $\Psi^{k}$. Let $\eta>0$ be such that (i) $\Psi(\lambda)<\lambda / 2$ for all $0<\lambda<\eta$, and (ii) the function $x \rightarrow \rho(0, x)$ takes all values in $(0, \eta)$. For each $x \in \mathbf{R}^{n}$ define 
$\tilde{\Psi}(x)=\Psi(\rho(0, x))$. Choose $x_{1}$ such that $0<\rho\left(0, x_{1}\right)<\eta, t_{1}=\tilde{\Psi}\left(x_{1}\right)$. Choose any $x_{2}$ such that $\rho\left(0, x_{2}\right)=\Psi\left(t_{1}\right)$ and put $t_{2}=\tilde{\Psi}\left(x_{2}\right)$. Continuing inductively we obtain a sequence $\left(x_{k}, t_{k}\right)$ such that $x_{k+1}$ satisfies $\rho\left(0, x_{k+1}\right)=\Psi^{2 k}\left(\rho\left(0, x_{1}\right)\right)=$ $\Psi\left(t_{k}\right)<t_{k} / 2$ and $t_{k+1}=\Psi^{2 k+1}\left(\rho\left(0, x_{1}\right)\right)=\Psi\left(\rho\left(0, x_{k+1}\right)\right)<\rho\left(0, x_{k+1}\right)<t_{k} / 2$ hence the sequence $\left\{t_{k}\right\}$ decreases to 0 . Thus the sequence satisfies the conditions of Proposition 1.12.

2. A Fatou theorem. In this section we use Theorem 1.11 to obtain a Fatoutype theorem for functions of the form $\int K(x, t, y) d \mu(y)$ where $\mu$ is a regular, Borel signed measure on $\mathbf{R}^{n}$ and the kernel $K$ satisfies certain general conditions. All measures will be regular, Borel.

For each $(x, t) \in \mathbf{R}_{+}^{n+1}$ and $y \in \mathbf{R}^{n}$ let $K(x, t ; y) \geq 0$ and satisfy:

(2.1) The function $u_{0}(x, t)=\int K(x, t ; y) d y$ approaches 1 continuously as $(x, t)$ $\rightarrow\left(x_{0}, 0\right)$ for each $x_{0} \in \mathbf{R}^{n}$.

(2.2) For all $(x, t) \in \mathbf{R}_{+}^{n+1}$,

$$
K(x, t ; y) \leq \frac{1}{|B(0, t)|} \cdot \varphi\left(\frac{\rho(x, y)}{t}\right),
$$

where $\varphi$ is a bounded, decreasing, real valued function on $[0, \infty)$ for which

$$
\sum_{k=1}^{\infty} \tau_{n}\left(2^{k+1}\right) \varphi\left(2^{k}\right)<\infty
$$

(2.4) For each $x_{0} \in \mathbf{R}^{n}$, open $W \ni x_{0}$ and $0<T \leq \infty$, there exist open sets $U \supset V \ni x_{0} . U \subset W$ and $\left(y_{0}, s_{0}\right) \in \mathbf{R}^{n} \times(0, T)$ such that for all $x \in V, y \in \mathbf{R}^{n} \backslash U$ and $t$ sufficiently close to 0 ,

$$
K(x, t ; y) \leq \delta(t) K\left(y_{0}, s_{0} ; y\right)
$$

where $\delta(t) \rightarrow 0$ as $t \rightarrow 0^{+}$.

For any signed measure $\mu$ on $\mathbf{R}^{n}$, let

$$
K \mu(x, t)=\int K(x, t ; y) d \mu(y)
$$

The following result is obtained by a standard dyadic decomposition argument (cf. [10, Lemma 4; 5, Lemma 7]).

LEMMA 2.5. Let $\Omega$ be any subset of $\mathbf{R}_{+}^{n+1}$ such that $(0,0) \in \bar{\Omega}$ and $\Omega(s) \subset \Omega(t)$ if $t>s$. For any measure $\mu$ on $\mathbf{R}^{n}$,

(a) $\sup _{(x, t) \in \Omega} K \mu\left(x_{0}+x, t\right) \leq C M_{\Omega} \mu\left(x_{0}\right)$,

(b) $\lim \sup _{\Omega \ni(x, t) \rightarrow 0} K \mu\left(x_{0}+x, t\right) \leq C M^{*} \mu\left(x_{0}\right)$, if $M_{\Omega} \mu\left(x_{0}\right)<\infty$.

ProOF. Let $B_{k}=B\left(x_{0}+x, 2^{k} t\right)$ for $k=0,1,2, \ldots$ Then

$$
K \mu\left(x_{0}+x, t\right)=\left(\int_{B_{0}}+\sum_{k=0}^{\infty} \int_{B_{k+1} \backslash B_{k}}\right) K\left(x_{0}+x, t ; y\right) d \mu(y) .
$$


For all $(x, t) \in \Omega$ and $k=0,1,2, \ldots$,

$$
\begin{aligned}
\int_{B_{0}} K\left(x_{0}+x, t ; y\right) d \mu(y) & \leq \varphi(0) \frac{\mu\left(B_{0}\right)}{\left|B_{0}\right|} \leq C M_{\Omega} \mu\left(x_{0}\right) \\
\int_{B_{k+1} \backslash B_{k}} K\left(x_{0}+x, t ; y\right) d \mu(y) & \leq \frac{1}{|B(0, t)|} \int_{B_{k+1} \backslash B_{k}} \varphi\left(\frac{\rho\left(x_{0}+x, y\right)}{t}\right) d \mu(y) \\
& \leq \varphi\left(2^{k}\right) \frac{\mu\left(B_{k+1}\right)}{|B(0, t)|} \\
& \leq C \varphi\left(2^{k}\right) \frac{\mu\left(B\left(x_{0}+x, 2^{k+1} t\right)\right)}{\left|B\left(0,2^{k+1} t\right)\right|} \cdot \tau_{n}\left(2^{k+1}\right) \\
& \leq C \varphi\left(2^{k}\right) \tau_{n}\left(2^{k+1}\right) M_{\Omega} \mu\left(x_{0}\right)
\end{aligned}
$$

and (a) follows. Part (b) follows by noting that, for all $(x, t) \in \Omega, K \mu\left(x_{0}+x, t\right)$ is majorized by a series whose $k$ th term is dominated by $\varphi\left(2^{k}\right) \tau_{n}\left(2^{k+1}\right) M_{\Omega} \mu\left(x_{0}\right)$, which is summable if $M_{\Omega} \mu\left(x_{0}\right)<\infty$.

THEOREM 2.6. If $\Omega$ is an $\alpha$-admissible subset of $\mathbf{R}_{+}^{n+1},(0,0) \in \bar{\Omega}$ and $\mu a$ finite signed measure on $\mathbf{R}^{n}$, then

$$
\lim _{\Omega \ni(x, t) \rightarrow 0} K \mu\left(x_{0}+x, t\right)=\frac{d \mu}{d m}\left(x_{0}\right) .
$$

PROOF.

$$
\begin{aligned}
\left|K \mu\left(x_{0}+x, t\right)-f\left(x_{0}\right)\right| & \leq\left|K \mu\left(x_{0}+x, t\right)-f\left(x_{0}\right) u_{0}(x, t)\right|+\left|u_{0}(x, t)-1\right|\left|f\left(x_{0}\right)\right| \\
& \leq \int K\left(x_{0}+x, t ; y\right) d \sigma(y)+\left|u_{0}(x, t)-1\right|\left|f\left(x_{0}\right)\right|
\end{aligned}
$$

where $\sigma=\left\|\mu-f\left(x_{0}\right) m\right\|$.

Let $x_{0}$ belong to the $\Omega$-Lebesgue set of $\mu$. Then by Theorem 1.11

$$
\lim _{\Omega \ni(x, t) \rightarrow 0} \frac{\sigma\left(B\left(x_{0}+x, t\right)\right)}{|B(0, t)|}=0 .
$$

Hence $M_{\Omega} \sigma\left(x_{0}\right)<\infty$ and so by the lemma and (2.1),

$$
\limsup _{\Omega \ni(x, t) \rightarrow 0}\left|K \mu\left(x_{0}+x, t\right)-f\left(x_{0}\right)\right| \leq C M_{\Omega}^{*} \sigma\left(x_{0}\right)=0 .
$$

Observe that property (2.4) of $K$ has not been used as yet. This property enables us to remove the finiteness condition on $\mu$ in the previous result.

LEMMA 2.7. Let $W \subset \mathbf{R}^{n}$ be an open set and $\mu$ a measure on $\mathbf{R}^{n}$ such that $K \mu(x, t)$ is finite on $\mathbf{R}^{n} \times(0, T)$ for some $0<T \leq \infty$. If $\mu(W)=0$ then $\lim _{(x, t) \rightarrow x_{0}} K \mu(x, t)=0$ for every $x_{0} \in W$.

ProOF. Let $x_{0} \in W$ and choose open sets $U, V$ and the point $\left(y_{0}, s_{0}\right) \in$ $\mathbf{R}^{n} \times(0, T)$ as in 2.4. Then for all $x \in V$ and $t$ sufficiently close to 0

$$
\begin{aligned}
K \mu(x, t) & =\int_{\mathbf{R}^{n} \backslash W} K(x, t ; y) d \mu(y)=\int_{\mathbf{R}^{n} \backslash U} K(x, t ; y) d \mu(y) \\
& \leq \delta(t) \int_{\mathbf{R}^{n}} K\left(y_{0}, s_{0} ; y\right) d \mu(y) \leq \delta(t) K \mu\left(y_{0}, s_{0}\right) .
\end{aligned}
$$

Our main result is an immediate consequence of this lemma and Theorem 2.6. 
THEOREM 2.8. Let $\Omega$ be an $\alpha$-admissible subset of $\mathbf{R}_{+}^{n+1},(0,0) \in \bar{\Omega}$ and $\mu a$ signed measure such that $K \mu(x, t)$ is finite on $\mathbf{R}^{n} \times(0, T)$ for some $0<T \leq \infty$. Then

$$
\lim _{\Omega \ni(x, t) \rightarrow 0} K \mu\left(x_{0}+x, t\right)=\frac{d \mu}{d m}\left(x_{0}\right) .
$$

3. Applications. In this section we use Theorem 2.8 to deduce a known Fatou theorem for positive solutions of the Laplace equation on $\mathbf{R}_{+}^{n+1}$ (cf. [10]) as well as analogues for parabolic equations.

The Laplace equation on an upper half-space. It is well-known that every positive solution, $u$, of Laplace's equation, $\Delta_{x} u+\partial^{2} u / \partial t^{2}=0$, on $\mathbf{R}_{+}^{n+1}$, where $x \in \mathbf{R}^{n}$, $t>0$, has the representation

$$
u(x, t)=c t+\int_{\mathbf{R}^{n}} P(x, t ; y) d \mu(y) .
$$

Here $\mu$ is a positive mesure on $\mathbf{R}^{n}$ and the Poisson kernel is

$$
P(x, t ; y)=P_{t}(x-y)=C_{n} t\left(|x-y|^{2}+t^{2}\right)^{-(n+1) / 2}, \quad C_{n}=\frac{\Gamma((n+1) / 2)}{\Pi^{(n+1) / 2}} .
$$

Let $\rho(x, y)=|x-y|$. Then the $\alpha$-standard region with vertex $y$ is the usual cone $\{(x, t):|x-y|<\alpha t\}$ of aperture $\alpha$ and vertex $y$. The $\alpha$-admissible subsets of $\mathbf{R}_{+}^{n+1}$ are those which satisfy the hypotheses of Theorem 1 in $[\mathbf{1 0}]$.

Clearly $P$ satisfies $(2.1)-(2.3)$ with $\varphi$ defined by $\varphi(\lambda)=\left(1+\lambda^{2}\right)^{-((n+1) / 2)}$. Now if $|x| \geq \alpha|y| \geq \beta$,

$$
\frac{P_{t}(x)}{P_{s}(y)}=\frac{t}{s}\left(\frac{s^{2}+|y|^{2}}{t^{2}+|x|^{2}}\right)^{(n+1) / 2} \leq \frac{t}{s}\left[\frac{s^{2}+|y|^{2}}{\alpha^{2}|y|^{2}}\right]^{(n+1) / 2} \leq \frac{t}{s}\left[\frac{s^{2}}{\beta^{2}}+\frac{1}{\alpha^{2}}\right]^{(n+1) / 2} .
$$

Hence in this case, $P_{t}(x) \leq C(s) t P_{s}(y)$.

Fix $x_{0} \in \mathbf{R}^{n}$, let $V=\left\{x:\left|x-x_{0}\right|<r\right\}, U=\left\{x:\left|x-x_{0}\right|<2 r\right\}, r>0$ sufficiently small. Then $x \in V, y \notin U \Rightarrow\left|x-x_{0}\right|<\frac{1}{2}\left|y-x_{0}\right|$. Thus $|x-y|>\frac{1}{2}\left|x_{0}-y\right| \geq r$. Hence for any $s>0, x \in V, y \notin U$,

$$
P(x, t ; y)=P_{t}(x-y) \leq C t P_{s}\left(x_{0}-y\right)=C t P\left(x_{0}, s ; y\right) \text { for all } t>0 .
$$

Hence $P$ satisfies (2.4) and we obtain the following result of Nagel and Stein [10].

THEOREM 3.1. Let $\Omega \subset \mathbf{R}_{+}^{n+1}$ be open $(0,0) \in \bar{\Omega}$ and satisfying

(i) there exists $A<\infty$ so that $|\Omega(t)| \leq A t^{n}$ for all $t>0$,

(ii) there exists $\alpha>0$ so that $(y, s) \in \Omega,|x-y|<\alpha(t-s) \Rightarrow(x, t) \in \Omega$.

Then for every signed measure $\mu$ on $\mathbf{R}^{n}$ such that $P_{t} * \mu(x)$ is finite on $\mathbf{R}_{+}^{n+1}$,

$$
\lim _{\Omega \ni(x, t) \rightarrow 0} P_{t} * \mu\left(x_{0}+x\right)=\frac{d \mu}{d m}\left(x_{0}\right) \text {. }
$$

Parabolic equations on an infinite slab. Let $X=\mathbf{R}^{n} \times(0, T)$ where $0<T \leq \infty$ is fixed. Let

$$
\begin{aligned}
L u= & \sum_{i, j=1}^{n} \frac{\partial}{\partial x_{j}}\left(A_{i j}(x, t) \frac{\partial u}{\partial x_{i}}+A_{j}(x, t) u\right) \\
& +\sum_{j=1}^{n} B_{j}(x, t) \frac{\partial u}{\partial x_{j}}+C(x, t) u-\frac{\partial u}{\partial t}
\end{aligned}
$$


be a second order linear parabolic operator in divergence form on $X$. Then under very general conditions on the coefficients (cf. [1] ), every positive (weak) solution of $L u=0$ on $X$ has the representation

$$
u(x, t)=\int_{\mathbf{R}^{n}} \Gamma(x, t ; y) d \mu(y),
$$

where $\mu$ is a measure on $\mathbf{R}^{n}$ and the fundamental solution, $\Gamma$, satisfies the condition that there are constants $p, p_{1}, p_{2}$ such that $p^{-1} W_{1} \leq \Gamma \leq p W_{2}$ on $X \times X$, where $W_{i}$ is the fundamental solution for the operator $p_{i} \Delta_{x}-\partial / \partial t$ (cf. [1]). That is,

$$
W_{i}(x, t ; y)=\left(4 p_{i} \Pi t\right)^{-n / 2} \exp \left[\frac{-|x-y|^{2}}{4 p_{i} t}\right], \quad t>0 .
$$

Let $\rho(x, y)=|x-y|^{2}$. The $\alpha$-standard region $A(y ; \alpha)$ with vertex $y$ is the parabolic region $\left\{(x, t):|x-y|^{2}<\alpha t\right\}$ of aperture $\alpha$, vertex $y$. An $\alpha$-admissible region $\Omega$ is one which satisfies

$$
(y, s) \in \Omega, \quad t>s \Rightarrow(y, t) \in \Omega:(0, t) \in \Omega \quad \text { for all } t>0
$$

and there exists an open $\Omega^{\prime} \supset \Omega$ such that

$$
\begin{aligned}
& \left|\Omega^{\prime}(t)\right| \leq C t^{n / 2} \quad \text { for all } t>0 \text { and for all }(y, s) \in \Omega, \\
& |x-y|^{2}<\alpha(t-s) \Rightarrow(x, t) \in \Omega^{\prime} .
\end{aligned}
$$

Property (2.1) is in Theorem 10 of $[\mathbf{1}]$. Choosing $\varphi(\lambda)=C \exp \left(-\lambda^{2} / 4 p_{2}\right)$, we see

$$
\Gamma(x, t ; y) \leq \frac{1}{t^{n / 2}} \varphi\left[\frac{\rho(x, y)}{t}\right] .
$$

Clearly $\varphi$ satisfies $(2.3)$ as $\tau_{n}(\lambda)=\lambda^{n / 2}$.

Now fix $x_{0} \in \mathbf{R}^{n}$ and let $V=\left\{x:\left|x-x_{0}\right|<r\right\}, U=\left\{x:\left|x-x_{0}\right|<2 r\right\}$ for $r$ sufficiently small. Then, as before, $x \in V, y \notin U \Rightarrow|x-y| \geq \frac{1}{2}\left|x_{0}-y\right| \geq r$. Hence for any $s>0, x \in V, y \notin U$,

$$
\begin{aligned}
\frac{\Gamma(x, t ; y)}{\Gamma\left(x_{0}, s ; y\right)} & \leq C\left(\frac{s}{t}\right)^{n / 2} \exp \left(\frac{\left|x_{0}-y\right|^{2}}{4 p_{1} s}-\frac{|x-y|^{2}}{4 p_{2} t}\right) \\
& =C\left(\frac{s}{t}\right)^{n / 2} \exp \left\{\left(\frac{t}{p_{1} s}-\frac{1}{4 p_{2}}\right) \frac{\left|x_{0}-y\right|^{2}}{4 t}\right\} \\
& \leq C\left(\frac{s}{t}\right)^{n / 2} \exp \left(-\frac{\left|x_{0}-y\right|^{2}}{32 p_{2} t}\right)
\end{aligned}
$$

and if $t$ is smaller than $p_{1} p / 8 p_{2}$,

$$
\leq C\left(\frac{s}{t}\right)^{n / 2} \exp \left(-\frac{r^{2}}{8 p_{2} t}\right) .
$$

Hence $\Gamma$ satisfies (2.4). We thus obtain the following result.

THEOREM 3.5. Let $\Omega \subset \mathbf{R}_{+}^{n+1}$ satisfy (3.2)-(3.4). Then for every signed measure $\mu$ on $\mathbf{R}^{n}$ for which $u(x, t)=\int \Gamma(x, t ; y) d \mu(y)$ is finite on $\mathbf{R}^{n} \times(0, T)$,

$$
\lim _{\Omega \ni(x, t) \rightarrow 0} u\left(x_{0}+x, t\right)=\frac{d \mu}{d m}\left(x_{0}\right) .
$$


Solutions of the heat equation as they approach a vertical boundary. Let $X=$ $\mathbf{R}^{n-1} \times \mathbf{R}_{+} \times(-\infty, T),-\infty<T \leq \infty$. Every positive solution, $u(x, t)$, of the heat equation $\Delta_{x} u=\partial u / \partial t$ on $X$, where $x=\left(x^{\prime}, x_{n}\right) \in \mathbf{R}^{n-1} \times \mathbf{R}_{+},-\infty<t<\tau$, can be written as

$$
\begin{aligned}
u(x, t)= & \int_{\mathbf{R}^{n-1} \times\{0\} \times(-\infty, T)} H_{b}(x, t) d \mu(b) \\
& +\int_{\mathbf{R}^{n-1}} x_{n} \exp \left(t\left|b^{\prime}\right|^{2}+\left\langle x^{\prime}, b^{\prime}\right\rangle\right) d \nu_{1}\left(b^{\prime}\right) \\
& +\int_{\mathbf{R}^{n-1} \times \mathbf{R}_{+}} \exp \left(t|b|^{2}+\left\langle x^{\prime}, b^{\prime}\right\rangle\right) \sinh \left(x_{n} b_{n}\right) d \nu_{2}\left(b^{\prime}, b_{n}\right),
\end{aligned}
$$

where $\langle$,$\rangle denotes the usual inner product and for b=\left(b^{\prime}, 0, s\right)$,

$$
H_{b}(x, t)= \begin{cases}(4 \Pi)^{-n / 2} \frac{x_{n}}{(t-s)^{(n+2) / 2}} \exp \left(-\frac{\left|x^{\prime}-b^{\prime}\right|^{2}+x_{n}^{2}}{4(t-s)}\right) & \text { if } t>s \\ 0 & \text { if } t \leq s\end{cases}
$$

(cf. $\left[\mathbf{7}, \S 4 ; \mathbf{9}\right.$, Corolary 3.3]). The vertical boundary is then $\mathbf{R}^{n-1} \times\{0\} \times(-\infty, T)$.

We show first that the last two integrals go to 0 continuously as $\left(x^{\prime}, x_{n}, t\right) \rightarrow$ $\left(y^{\prime}, 0, s\right)$. Let $v_{1}(x, t)$ be the first of these and $v_{2}(x, t)$ the last. For all $(x, t)$ such $\left|x^{\prime}-y^{\prime}\right|<\delta, x_{n}<\delta, t<t_{0}<t_{0}+\delta=t_{1}<T$,

$$
\begin{aligned}
\exp \left(t\left|b^{\prime}\right|^{2}+\left\langle x^{\prime}, b^{\prime}\right\rangle\right) & \leq \exp \left(t\left|b^{\prime}\right|^{2}+\left\langle y^{\prime}, b^{\prime}\right\rangle+\left|x^{\prime}-y^{\prime}\right|\left|b^{\prime}\right|\right) \\
& \leq \exp \left(t\left|b^{\prime}\right|^{2}+\left\langle y^{\prime}, b^{\prime}\right\rangle+\delta\left|b^{\prime}\right|\right) \\
& \leq C \exp \left(t_{1}\left|b^{\prime}\right|^{2}+\left\langle y^{\prime}, b^{\prime}\right\rangle\right) .
\end{aligned}
$$

Thus $v_{1}(x, t) \leq C x_{n} v_{1}\left(\left(y^{\prime}, 1\right), t_{1}\right) \rightarrow 0$. As

$$
v_{2}(x, t) \leq C \int_{\mathbf{R}^{n-1} \times \mathbf{R}_{+}} \exp \left(t_{1}|b|^{2}+\left\langle y^{\prime}, b^{\prime}\right\rangle\right) \sinh \left(x_{n} b_{n}\right) d \nu_{2}(b),
$$

and the integrand is dominated by the $\nu_{2}$-integrable $\exp \left(t_{1}|b|^{2}+\left\langle y^{\prime}, b^{\prime}\right\rangle\right) \sinh \delta b_{n}$ (its integral is $\left.v_{2}\left(\left(y^{\prime}, \delta\right), t_{1}\right)\right), v_{2}(x, t) \rightarrow 0$ by Lebesgue's Dominated Convergence Theorem.

Now consider solutions of the form $\int H_{b}(x, t) d \mu(b)$. To make this amenable to the notations established in this paper, we make a slight change in notation. For each $(x, t) \in \mathbf{R}^{n} \times \mathbf{R}_{+}, y=\left(y^{\prime}, y_{n}\right) \in \mathbf{R}^{n-1} \times \mathbf{R}$, define

$$
K(x, t, y)= \begin{cases}(4 \Pi)^{-n / 2} t\left(x_{n}-y_{n}\right)^{-(n+2) / 2} \exp \left(-\frac{\left|x^{\prime}-y^{\prime}\right|^{2}+t^{2}}{4\left(x_{n}-y_{n}\right)}\right) & \text { if } x_{n}>y_{n} \\ 0 & \text { if } x_{n} \leq y_{n}\end{cases}
$$

The problem now becomes one of examining $K \mu(x, t), x \in \mathbf{R}^{n-1} \times(-\infty, T), t>0$, as $(x, t) \rightarrow\left(x_{0}, 0\right)$. $K \mu(x, t)$ satisfies the heat equation

$$
\sum_{i=1}^{n-1} \frac{\partial^{2} u}{\partial x_{i}^{2}}+\frac{\partial^{2} u}{\partial t^{2}}=\frac{\partial u}{\partial x_{n}}
$$


For each $x, y \in \mathbf{R}^{n}$ define $\rho(x, y)=\left(\left|x^{\prime}-y^{\prime}\right|^{2}+\left|x_{n}-y_{n}\right|\right)^{1 / 2}$. To see that $\rho$ is a metric, observe that

$$
\begin{aligned}
\left|x^{\prime}-y^{\prime}\right|^{2}+\left|x_{n}-y_{n}\right| \leq\left|x^{\prime}\right|^{2}+\left|x_{n}\right|+\left|y^{\prime}\right|^{2}+\left|y_{n}\right|-2\left\langle x^{\prime}, y^{\prime}\right\rangle \\
\leq\left|x^{\prime}\right|^{2}+\left|x_{n}\right|+\left|y^{\prime}\right|^{2}+\left|y_{n}\right|+2\left(\left|x^{\prime}\right|^{2}+\left|x_{n}\right|\right)^{1 / 2}\left(\left|y^{\prime}\right|^{2}+\left|y_{n}\right|\right)^{1 / 2} \\
=\left[\left(\left|x^{\prime}\right|^{2}+\left|x_{n}\right|\right)^{1 / 2}+\left(\left|y^{\prime}\right|^{2}+\left|y_{n}\right|\right)^{1 / 2}\right]^{2} .
\end{aligned}
$$

Hence $\rho(x, y) \leq \rho(x, 0)+\rho(y, 0)$, which, by translation invariance, implies the triangle inequality.

The $\alpha$-standard region $A(y ; \alpha)$ is $\left\{(x, t):\left(\left|x^{\prime}-y^{\prime}\right|^{2}+\left|x_{n}-y_{n}\right|\right)^{1 / 2}<\alpha t\right\}$. This corresponds exactly to the "parabolic cones" defined in [12]. Two other definitions of two-sided-parabolic regions have been studied in the literature. In our notation they would take the form

$$
\Gamma(y ; \alpha)=\left\{(x, t):\left|x^{\prime}-y^{\prime}\right|+\left|x_{n}-y_{n}\right|^{1 / 2}<\alpha t\right\}
$$

defined in $[6]$ and

$$
T P(y ; \alpha: \beta)=\left\{(x, t):\left|x_{n}-y_{n}\right|<\alpha\left(\left|x^{\prime}-y^{\prime}\right|^{2}+t^{2}\right), t>\beta\left|x^{\prime}-y^{\prime}\right|\right\}
$$

defined in $[8]$. It is obvious that these regions are all equivalent to the $\alpha$-standard regions, since, for each $\alpha, \beta>0$

$$
\Gamma(y, \alpha) \subset A(y, \alpha) \subset \Gamma(y, \alpha \sqrt{2})
$$

and

$$
A(y, \alpha) \subset T P\left(y ; \alpha^{2}: \alpha^{-1}\right) ; T P(y ; \alpha: \beta) \subset A(y, C) .
$$

An $\alpha$-admissible region $\Omega$ is one which satisfies

$$
\begin{aligned}
& (0,0) \in \bar{\Omega}, \\
& |\Omega(t)| \leq C t^{n+1} \quad \text { for all } t>0, \\
& (y, s) \in \Omega, \quad\left(\left|x^{\prime}-y^{\prime}\right|^{2}+\left|x_{n}-y_{n}\right|\right)^{1 / 2}<\alpha(t-s) \Rightarrow(x, t) \in \Omega .
\end{aligned}
$$

We now verify the conditions on $K$ in $\S 2$. Simple calculations give $\int K(x, t ; y) d y$ $=1$ and

$$
K(x, t ; y) \leq \frac{C}{t^{n+1}}\left\{\frac{1}{(\rho(x, y) / t)^{2}+1}\right\}^{(n+2) / 2}
$$

for all $(x, t)$. Choosing $\varphi(\lambda)=\left(\lambda^{2}+1\right)^{-(n+2) / 2}$, we see that $(2.1)-(2.3)$ are satisfied. The following lemma verifies (2.4).

LemMA 3.10. Fix $r, s>0, a>2 r, y_{0}=(0, a) \in \mathbf{R}^{n}$. Then $K(x, t ; y) \leq$ $C t K\left(y_{0}, s ; y\right)$ if $\left|x^{\prime}\right| \leq r,\left|x_{n}\right| \leq r$ and either $\left|y^{\prime}\right| \geq 4 r$ or $\left|y_{n}\right| \geq 2 r$.

ProOF. We only need to consider $x_{n}>y_{n}$. Then $a>r \geq x_{n}$. Hence,

$$
\frac{K(x, t ; y)}{K\left(y_{0}, s ; y\right)}=\left(\frac{t}{s}\right)\left(\frac{a-y_{n}}{x_{n}-y_{n}}\right)^{(n+2) / 2} \exp \left\{\frac{\left|y^{\prime}\right|^{2}+s^{2}}{4\left(a-y_{n}\right)}-\frac{\left|x^{\prime}-y^{\prime}\right|^{2}+t^{2}}{4\left(x_{n}-y_{n}\right)}\right\} \text {. }
$$


Now,

$$
\begin{aligned}
\frac{\left|y^{\prime}\right|^{2}}{a-y_{n}}-\frac{\left|x^{\prime}-y^{\prime}\right|^{2}}{x_{n}-y_{n}}= & \frac{\left(x_{n}-y_{n}\right)\left|y^{\prime}\right|^{2}-\left(a-y_{n}\right)\left[\left|x^{\prime}\right|^{2}-2\left\langle x^{\prime}, y^{\prime}\right\rangle+\left|y^{\prime}\right|^{2}\right]}{\left(a-y_{n}\right)\left(x_{n}-y_{n}\right)} \\
= & \frac{\left(x_{n}-a\right)\left|y^{\prime}\right|^{2}+2\left(a-y_{n}\right)\left\langle x^{\prime}, y^{\prime}\right\rangle-\left(a-y_{n}\right)\left|x^{\prime}\right|^{2}}{\left(a-y_{n}\right)\left(x_{n}-y_{n}\right)} \cdot\left(\frac{a-x_{n}}{a-x_{n}}\right) \\
= & -\frac{\left|\left(a-x_{n}\right) y^{\prime}-\left(a-y_{n}\right) x^{\prime}\right|^{2}}{\left(a-x_{n}\right)\left(a-y_{n}\right)\left(x_{n}-y_{n}\right)} \\
& +\frac{\left(\left(a-y_{n}\right)^{2}-\left(a-y_{n}\right)\left(a-x_{n}\right)\right)\left|x^{\prime}\right|^{2}}{\left(a-x_{n}\right)\left(a-y_{n}\right)\left(x_{n}-y_{n}\right)} \\
= & -\frac{\left|\left(a-x_{n}\right) y^{\prime}-\left(a-y_{n}\right) x^{\prime}\right|^{2}}{\left(a-x_{n}\right)\left(a-y_{n}\right)\left(x_{n}-y_{n}\right)}+\frac{\left|x^{\prime}\right|^{2}}{a-x_{n}} .
\end{aligned}
$$

Therefore

$$
\begin{aligned}
\frac{K(x, t ; y)}{K\left(y_{0}, s ; y\right)}= & \frac{t}{s} \cdot\left(\frac{a-y_{n}}{x_{n}-y_{n}}\right)^{(n+2) / 2} \\
& \cdot \exp \left\{-\frac{\left|\left(a-x_{n}\right) y^{\prime}-\left(a-y_{n}\right) x^{\prime}\right|^{2}}{4\left(a-x_{n}\right)\left(a-y_{n}\right)\left(x_{n}-y_{n}\right)}\right. \\
& \left.\quad+\frac{\left|x^{\prime}\right|^{2}}{4\left(a-x_{n}\right)}+\frac{s^{2}}{4\left(a-y_{n}\right)}-\frac{t^{2}}{4\left(x_{n}-y_{n}\right)}\right\} \\
\leq & C t\left(\frac{a-y_{n}}{x_{n}-y_{n}}\right)^{(n+2) / 2} \\
& \cdot \exp \left\{-\frac{\left|\left(a-x_{n}\right) y^{\prime}-\left(a-y_{n}\right) x^{\prime}\right|^{2}}{4\left(a-x_{n}\right)\left(a-y_{n}\right)\left(x_{n}-y_{n}\right)}+\frac{s^{2}}{4\left(a-y_{n}\right)}\right\} .
\end{aligned}
$$

If $\left|y_{n}\right| \geq 2 r$, since $y_{n}<x_{n} \leq r, y_{n} \leq-2 r$. Hence $\left(a-y_{n}\right) /\left(x_{n}-y_{n}\right)$ and $1 /\left(a-y_{n}\right)$ are bounded above and so $K(x, t ; y) \leq C t K\left(y_{0}, s ; y\right)$.

Now, if $\left|y^{\prime}\right| \geq 4 r$ and $\left|y_{n}\right|<2 r$,

$$
\begin{aligned}
K(x, t ; y) & \leq C t\left(\frac{\left(a-y_{n}\right)^{2}\left(a-x_{n}\right)}{\left|\left(a-x_{n}\right) y^{\prime}-\left(a-y_{n}\right) x^{\prime}\right|^{2}}\right)^{(n+2) / 2} \exp \left(\frac{s^{2}}{4\left(a-y_{n}\right)}\right) K\left(y_{0}, s ; y\right) \\
& \leq C t\left|\frac{y^{\prime}}{a-y_{n}}-\frac{x^{\prime}}{a-x_{n}}\right|^{-(n+2)} K\left(y_{0}, s ; y\right)
\end{aligned}
$$

since $a-y_{n}>a-2 r$ and $a-x_{n}>r$. Finally

$$
\left|\frac{y^{\prime}}{a-y_{n}}-\frac{x^{\prime}}{a-x_{n}}\right| \geq \frac{4 r}{a+2 r}-\frac{r}{a-r}
$$

so in this case $K(x, t ; y) \leq C t K\left(y_{0}, s ; y\right)$ and the lemma is proved.

THEOREM 3.11. Let $\Omega \subset \mathbf{R}_{+}^{n+1}$ be open, $(0,0) \in \bar{\Omega}$ and satisfy (3.8) and (3.9). Then for every signed measure $\mu$ on $\mathbf{R}^{n+1} \times(-\infty, T)$ such that $K \mu(x, t)<\infty$ on $\mathbf{R}^{n-1} \times(-\infty, T) \times \mathbf{R}_{+}$,

$$
\lim _{\Omega \ni(x, t) \rightarrow 0} K \mu\left(x_{0}+x, t\right)=\frac{d \mu}{d m}\left(x_{0}\right) .
$$

This is obtained by simply extending $\mu$ to be zero off $\mathbf{R}^{n-1} \times(-\infty, T)$. 
In the usual notation for the heat equation $\Delta_{x} u=\partial u / \partial t$ on

$$
X=\left\{\left(x^{\prime}, x_{n}, t\right): x^{\prime} \in \mathbf{R}^{n-1}, x_{n}>0, t<T\right\}
$$

we have obtained the following result.

THEOREM 3.12. Let $\Omega \subset X$ have $(0,0)$ as a limit point and satisfy:

(i) $\left|\left\{\left(x^{\prime}, 0, t\right):\left(x^{\prime}, x_{n}, t\right) \in \Omega\right\}\right| \leq C x_{n}^{n+1}$ for all $x_{n}>0$,

(ii) $(y, s) \in \Omega,\left(\left|x^{\prime}-y^{\prime}\right|^{2}+|t-s|\right)^{1 / 2}<\alpha\left(x_{n}-y_{n}\right) \Rightarrow(x, t) \in \Omega$.

Then for signed measures $\mu, \nu_{1}, \nu_{2}$ and $u(x, t)$ as in (3.6),

$$
\lim _{\Omega \ni(x, t) \rightarrow 0} u\left(x_{0}+x, t\right)=\frac{d \mu}{d m}\left(x_{0}\right) .
$$

4. A converse. Let $\Omega \subset \mathbf{R}_{+}^{n+1}$ and let $K$ be as in $\S 2$. Define, for each $f \in L^{1}\left(\mathbf{R}^{n}\right)$ and $x_{0} \in \mathbf{R}^{n}$,

$$
M_{\Omega}^{K} f\left(x_{0}\right)=\sup _{(x, t) \in \Omega} K|f|\left(x_{0}+x, t\right)
$$

Theorem 1.8 and Lemma 2.5 imply $M_{\Omega}^{K}$ is weak-type $(1,1)$ in case $\Omega$ is $\alpha$-admissible. We wish now to show that the $\alpha$-admissible condition is necessary in the sense that if $M_{\Omega}^{K}$ is weak-type $(1,1)$, then there is an $\alpha$-admissible set, $\Omega_{\alpha}$, containing $\Omega$. For this purpose we impose the following additional restrictions on $K$ :

(4.1) There is a $C>0$ independent of $t$ such that $\int_{B(0, t)} K(0, t ; y) d y>C$

(4.2) (Semigroup property) For all $s, t>0$ and $x, z \in \mathbf{R}^{n}$

$$
\int K(y, s ; x) K(z, t ; y) d y=K(z, s+t ; y) \text {. }
$$

(4.3) For all $t>0, x \in \mathbf{R}^{n}, y \in \mathbf{R}^{n}, K(x, t ; y)=K(x-y, t ; 0)$.

These conditions hold for the kernels associated with the Laplace and heat equations considered in $\S 3$. Indeed, that (4.1) holds for the first two is obvious. Put now $E=\left\{\left(y^{\prime}, y_{n}\right):\left|y^{\prime}\right|^{2}+y_{n}<t^{2}, y_{n}>0\right\}$. Then

$$
\begin{aligned}
\int_{B(0, t)} K(0, t ; y) d y & =C \int_{E} \frac{t}{y_{n}^{(n+2) / 2}} \exp \left(-\frac{\left|y^{\prime}\right|^{2}+y_{n}+t^{2}}{4 y_{n}}\right) d y^{\prime} d y_{n} \\
& \geq C \int_{E} \frac{t}{y_{n}^{(n+2) / 2}} \exp \left(-\frac{t^{2}}{2 y_{n}}\right) d y^{\prime} d y_{n} \\
& =C \int_{0}^{t^{2}} \frac{t}{y_{n}^{(n+2) / 2}} \exp \left(\frac{-t^{2}}{2 y_{n}}\right)\left(t^{2}-y_{n}\right)^{(n-1) / 2} d y_{n} \\
& =C \int_{1}^{\infty} s^{-1 / 2} e^{-s}(s-1)^{(n-1) / 2} d s .
\end{aligned}
$$

For the semigroup property see $[\mathbf{1 1}, \mathbf{1}$ and $\mathbf{7}]$.

Let $\alpha>0$. Put

$$
\Omega_{\alpha}=\left\{(x, t) \in \mathbf{R}_{+}^{n+1}: \rho\left(x, x_{0}\right)<\alpha\left(t-t_{0}\right) \text { for some }\left(x_{0}, t_{0}\right) \in \Omega\right\} .
$$

As in Proposition 1.12, $\Omega_{\alpha}$ satisfies (2)(c)(ii) of Definition 1.3 (take $\Omega_{\alpha}^{\prime}=\Omega_{\gamma \alpha}$ ). 
THEOREM 4.4. Suppose $M_{\Omega}^{K}$ is weak-type $(1,1)$, that is there exists $C>0$ independent of $f \in L^{1}\left(\mathbf{R}^{n}\right)$ such that for every $\lambda>0$,

$$
\left|\left\{x \in \mathbf{R}^{n}: M_{\Omega}^{K} f(x)>\lambda\right\}\right| \leq C\|f\|_{1} / \lambda .
$$

Then $\Omega_{\alpha}$ is $\alpha$-admissible for every $\alpha>0$.

PROOF. Let $g \in L^{p}\left(\mathbf{R}^{n}\right)$ for $p>1$. For any $(x, t) \in \Omega_{\gamma \alpha}$ and $\left(x_{0}, t_{0}\right)$ a corresponding point of $\Omega$

$$
\begin{aligned}
K g(x+w, t) & =\int K(x+w, t ; y) g(y) d y \\
& =\int g(y) d y \int K\left(x+w, t-t_{0} ; z\right) K\left(z, t_{0} ; y\right) d z \\
& =\int K\left(x+w, t-t_{0} ; z\right) d z \int g(y) K\left(z-x_{0}+x_{0}, t_{0} ; y\right) d y \\
& \leq \int M_{\Omega}^{K} g(z) K\left(x-x_{0}+w, t-t_{0} ; z\right) d z \\
& \leq M_{A(0, \gamma \alpha)}^{K}\left(M_{\Omega}^{K} g\right)(w) .
\end{aligned}
$$

Since $(x, t)$ is an arbitrary point of $\Omega_{\gamma \alpha}$,

$$
M_{\Omega_{\gamma \alpha}}^{K} g(w) \leq M_{A(0 ; \gamma \alpha)}^{K}\left(M_{\Omega}^{K} g\right)(w) .
$$

By the Marcinkiewicz Interpolation Theorem and Theorem 1.8 applied to $A(0 ; \gamma \alpha)$,

$$
\left\|M_{\Omega_{\gamma \alpha}}^{K} g\right\|_{p} \leq C\|g\|_{p} .
$$

It follows that $M_{\Omega_{\gamma \alpha}}^{K}$ is weak type $(p, p)$ for every $p>1$.

Let $t>0$. Put $g(y)=|B(0, t)|^{-1 / p}$ if $\rho(0, y)<t$ and 0 otherwise. Let $(x, t) \in$ $\Omega_{\gamma \alpha}$. Then

$$
\begin{aligned}
M_{\Omega_{\gamma \alpha}}^{K} g(-x) & \geq \int K(0, t ; y) g(y) d y \\
& =\int_{B(0, t)} K(0, t ; y)|B(0, t)|^{-1 / p} d y>C|B(0, t)|^{-1 / p}
\end{aligned}
$$

Hence

$$
\begin{aligned}
\left|\left\{x:(x, t) \in \Omega_{\gamma \alpha}\right\}\right| & \leq\left|\left\{x: M_{\Omega_{\gamma \alpha}}^{K} g(-x) \geq C|B(0, t)|^{-1 / p}\right\}\right| \\
& \leq C|B(0, t)| .
\end{aligned}
$$

Thus $\Omega_{\alpha}$ is $\alpha$-admissible and we are done.

\section{REFERENCES}

1. D. G. Aronson, Non-negative solutions of linear parabolic equations, Ann. Scuola Norm. Sup. Pisa 22 (1968), 607-694.

2. J. Chabrowski, Private communication.

3. Ronald Coifman and Guido Weiss, Analyse harmonique non-commutative sur certaines espaces homogènes, Lecture Notes in Math., vol. 242, Springer-Verlag, New York and Berlin, 1971.

4. J. L. Doob, Relative limit theorems in analysis, J. Analyse Math. 8 (1960/61), 289-306.

5. Raymond Johnson, Representation theorems and Fatou theorems for second-order linear parabolic partial differential equations, Proc. London Math. Soc. (3) 23 (1971), 325-347. 
6. J. T. Kemper, Temperatures in several varaibles: kernel functions representations, and parabolic boundary values, Trans. Amer. Math. Soc. 167 (1972), 243-262.

7. B. A. Mair, Integral representations for positive solutions of the heat equation on some unbounded domains, Illinois J. Math. 30 (1986), 175-184.

8. __ Boundary behavior of positive solutions of the heat equation on a semi-infinite slab, Trans. Amer. Math. Soc. 295 (1986), 687-697.

9. B. A. Mair and J. C. Taylor, Integral representation of positive solutions of the heat equation, Lecture Notes in Math., vol. 1096, Springer-Verlag, New York and Berlin, 1984, pp. 419-433.

10. Alexander Nagel and Elias M. Stein, On certain maximal functions and approach regions, Adv. in Math. 54 (1984), 83-106.

11. Elias M. Stein, Singular integrals and differentiability properties of functions, Princeton Univ. Press, Princeton, N.J., 1970.

12. C. C. Tu, Non-tangential limits of a solution of a boundary-value problem for the heat equation, Math. Systems Theory 3 (1969), 130-138.

Department of Mathematics, Texas TeCh University, Lubbock, Texas 79409

Department of Mathematics, The Pennsylvania State University, Delaware County Campus, 25 Yearsley Mill Road, Media, Pennsylvania 19063 